\title{
Effets thermiques dans la cavitation des fluides cryogéniques
}

\author{
D.H. Fruman et F. Beuzelin \\ Groupe Phénomènes d'Interface \\ Ecole Nationale Supérieure de Techniques Avancées \\ Palaiseau, France
}

\section{Nomenclature}

$B \quad$ largeur de la veine d'essais

$C_{f}$ coefficient de frottement local

$C_{p} \quad$ chaleur spécifique à pression constante

$C_{Q} \quad$ coefficient de débit

$E_{v} \quad$ épaisseur équivalente de la cavité $\left(=C_{Q} l\right)$

$l \quad$ longueur de la cavité

$L \quad$ chaleur latente de vaporisation

$P \quad$ nombre de Prandtl

$p_{0} \quad$ pression de l'écoulement à l'entrée

$p_{c} \quad$ pression de vapeur à la température $T_{c}$

$p_{v}$ pression de vapeur à la température $T_{0}$

$Q_{v} \quad$ débit volumique de vapeur produite (égal au débit d'air injecté)

$\mathrm{Re}_{5}$ nombre de Reynolds calculé avec l'épaisseur de la couche limite

$R_{l} \quad$ nombre de Reynolds relatif à la longueur de la plaque

$R_{x} \quad$ nombre de Reynolds relatif à l'abscisse le long de la plaque
$T_{c} \quad$ température de la vapeur dans la cavité

$T_{0} \quad$ température du liquide à l'entrée

$v_{0} \quad$ vitesse de l'écoulement à l'entrée

$v_{g} \quad$ vitesse débitante d'air au travers de la surface de la cavité

$\delta \quad$ épaisseur de la couche limite

$\delta_{b} \quad$ épaisseur du film visqueux

$\Delta \sigma \quad$ écart du nombre de cavitation $\left(\sigma_{c}-\sigma_{v}\right)$

$\Delta T$ écart de température $\left(T_{0}-T_{c}\right)$

$\varepsilon \quad$ rugosité apparente de l'interface

$\Phi_{p} \quad$ flux thermique à la paroi (à l'interface de la cavité)

$\eta \quad$ facteur d'efficacité

$\lambda$ conductibilité thermique du liquide

$\mu \quad$ viscosité du liquide

$\rho_{v} \quad$ masse spécifique de la vapeur

$\rho \quad$ masse spécifique du liquide

$\sigma_{c} \quad$ nombre de cavitation calculé avec la pression dans la cavité

$\sigma_{v}$ nombre de cavitation calculé avec la pression de vapeur à la température $\mathrm{T}_{0}$

\section{Thermal effects in the cavitation of cryogenic fluids}

The vapour production required to sustain a cavity leads to a temperature reduction at the interface. This temperature reduction, though quite small in the case of water, is significant in the case of cryogenic fluids. A method assuming that (i) the vapor production is equal to the volume flow rate of air required to sustain a ventilated cavity and (ii) that heat transfer at the interface is analogous to that over a rough flat plate, gives reasonable estimates of the temperature reduction. 


\section{Introduction}

Il est bien connu que la similitude entre deux écoulements cavitants s'établit si, à géométrie semblable, l'on réalise l'égalité des nombres sans dimension les caractérisant et, en particulier, celui de cavitation, $\sigma_{c}$, défini par,

$$
\sigma_{c}=\frac{2\left(p_{0}-p_{c}\right)}{\rho V_{0}^{2}}=\frac{2\left(p_{0}-p_{v}\right)}{\rho V_{0}^{2}}+\frac{2\left(p_{v}-p_{c}\right)}{\rho V_{0}^{2}}=\sigma_{v}+\Delta \sigma
$$

où $p_{0}$ représente la pression à l'entrée, $p_{c}$ celle dans la poche à la température $T_{c}, p_{v}$ celle de la vapeur à la température d'entrée $T_{0}$ et $V_{0}$ est la vitesse d'entrée. La valeur de $\sigma_{v}$ est calculée à partir des conditions d'entrée quels que soient l'écoulement et le fluide considérés. Cependant, pour déterminer $\Delta \sigma$ il faut connaître l'écart de température $\Delta T=T_{0}-T_{c}$, qui est négligeable dans le cas de l'eau froide et dans bien d'autres fluides opérant loin des conditions critiques, mais qui devient significatif dans le cas des fluides cryogéniques.

La prise en compte de ce que l'on convient d'appeler les effets thermiques dans la cavitation a fait l'objet de multiples tentatives depuis le travail de STAHL et STEPANOFF (1956) suivi de ceux de MOORE et RUGGERI (1969), de HORD (1972) et de Holl et al. (1975). L'approche choisie par ces auteurs a consisté essentiellement dans l'établissement, à partir de résultats expérimentaux obtenus avec des fluides présentant des effets thermiques dans différentes géométries, de corrélations faisant intervenir le produit de puissances de nombres sans dimension. Dans cette étude, on propose une méthode semi-empirique basée sur les hypothèses que, d'une part, le débit de vapeur à produire est égal à celui d'air nécessaire pour entretenir une cavité ventilée et que, d'autre part, l'échange de chaleur à l'interface est analogue à celui existant sur une plaque plane rugueuse. Cette méthode permet d'évaluer $\Delta T$ pour une poche de cavitation partielle bidimensionnelle de largeur $B$ et de longueur $l$ en fonction des conditions d'entrée $\left(T_{0}, V_{0}\right.$ et $\left.P_{0}\right)$. Les résultats sont comparés à ceux de HoRD pour le cas d'un venturi (1972a) et d'un profil (1972b).

\section{Modélisation}

Puisque la pression à l'intérieur de la cavité peut être supposée constante et que la courbure de l'interface est faible, cette dernière peut être assimilée à une plaque plane sans gradient de pression. La poche est stable, il y a donc équilibre entre l'apport de vapeur par vaporisation et l'entraînement de vapeur par arrachement. La vaporisation nécessite un apport continu de chaleur cédée par le liquide à l'interface. On impose donc sur la plaque un flux de chaleur uniformément réparti, $\Phi_{p}$, que l'on peut calculer si l'on connaît le débit volumique $Q_{v}$ de vapeur produite :

$$
\Phi_{p}=\frac{L \rho_{v} Q_{v}}{B l}
$$

où $L$ et $\rho_{v}$ sont respectivement la chaleur latente de vaporisation et la masse volumique de la vapeur. On suppose que $Q_{v}$ peut être obtenu à partir d'expériences réalisées sur des cavités ventilées en admettant que, pour une géométrie d'obstacle donnée, il est égal au débit volumique de gaz entraîné et dépend de $l$ et de $V_{0}$ (mais pas de la nature du gaz, vapeur ou gaz non condensable, ni de la nature du liquide).

Pour estimer le flux de chaleur on considère les couches limites dynamique et thermique sur une plaque plane pour des nombres de Prandtl, $P$, voisins de 1 et des nombres de Reynolds relatifs à la longueur de la poche de l'ordre de $10^{6}$ (valeurs qui correspondent aux essais de Hord). La couche limite est alors constituée de (BRUN (1970) p. 194 et suivantes) :

i) un film visqueux, à la traversée duquel le flux de chaleur, $\Phi$, et la contrainte visqueuse, $\tau$, sont constants et dont le rapport à la paroi (indice $p$ ) est donné par :

$$
\frac{\Phi_{p}}{\tau_{p}}=-\frac{\lambda}{\mu} \frac{T_{b}-T_{p}}{V_{b}}
$$

où $\lambda$ et $\mu$ sont respectivement la conductibilité thermique et la viscosité du liquide et l'indice $b$ concerne des quantités à la limite de la couche visqueuse, et

ii) une partie turbulente dans laquelle l'analogie de Reynolds permet d'écrire, au point de raccordement de celle-ci et du film visqueux,

$$
\frac{\Phi_{b}}{\tau_{b}}=-C_{p} \frac{T_{0}-T_{b}}{V_{0}-V_{b}}
$$

où $C_{p}$ est la chaleur spécifique à pression constante. En opérant sur ces deux expressions sachant que $\frac{\Phi_{p}}{\tau_{p}}=\frac{\Phi_{b}}{\tau_{b}}$ on obtient,

$$
T_{0}-T_{p}=\frac{\Phi_{p} V_{0}}{\tau_{p} C_{p}}\left[1+(P-1) \frac{V_{b}}{V_{0}}\right] .
$$

Le rapport des épaisseurs de la couche limite, $\delta$, et du film visqueux, $\delta_{b}$, est donné dans la partie turbulente par :

$$
\frac{\delta_{b}}{\delta}=\left(\frac{V_{b}}{V_{0}}\right)^{7}
$$

et, dans la couche visqueuse par:

$$
\frac{\delta_{b}}{\delta}=\left(\frac{V_{b}}{V_{0}}\right) \frac{2}{C_{f} \operatorname{Re}_{8}}
$$

où $C_{f}$, le coefficient de frottement local, et $\mathrm{Re}_{\delta}$, le nombre de Reynolds calculé avec l'épaisseur de la couche limite valent dans le cas d'une plaque plane lisse et d'un profil de vitesse en puissance $1 / 7$, $C_{f}=0,0464 \mathrm{Re}_{\delta}^{-0.25}=0,0592 R_{x}^{-0,2}$ et $\mathrm{Re}_{5}=0,38 R_{x}^{0.8}$. En égalisant le rapport des épaisseurs de couches limites et en remplaçant $C_{f}$ et $\mathrm{Re}_{8}$ par les expressions ci-dessus on obtient,

$$
\frac{V_{b}}{V_{0}}=2,11 R_{x}^{-0,1}
$$

avec $R_{x}$ le nombre de Reynolds relatif à l'abscisse le long de la plaque. Le problème se trouve alors résolu pour une 
plaque plane lisse, si l'on combine les équations (2) et (3) avec le $\tau_{p}$ issu du coefficient de frottement donné cidessus.

A égalité de flux, l'écart de température pour une plaque plane rugueuse est égal à celui sur une plaque lisse multiplié par l'inverse du rapport de leur coefficient de frottement respectif affecté d'un facteur d'efficacité $\eta$ qui dépend de la hauteur de la rugosité et du nombre de Prandtl (ECKERT et DRAKE (1972)). Avec les indices $r$ et $l$ pour indiquer qu'il s'agit de la plaque plane rugueuse et lisse respectivement on a en posant $\Delta T=T_{0}-T_{p}$,

$$
\Delta T_{r}=\frac{\Delta T_{l}}{\eta} \frac{C_{f l}}{C_{f r}} .
$$

En combinant les équations (2), (3) et (4), avec $\eta \approx 1$ pour le cas qui nous occupe, et en supposant que l'épaisseur des rugosités, $\varepsilon$, est beaucoup plus grande que celle de la couche visqueuse de sorte que le coefficient de frottement soit indépendant du nombre de Reynolds,

$$
C_{f r}=0,0139\left(\frac{x}{\varepsilon}\right)^{-\frac{1}{7}}
$$

on obtient l'écart de température sur paroi rugueuse dont il sera question plus loin.

Pour pouvoir calculer l'écart de température il faut connaître le débit volumique de vapeur produite, qui est assimilé à celui d'entraînement de gaz non condensable d'une cavité ventilée. Des expériences destinées à mesurer ce dernier pour des poches ventilées stables, derrière une marche et en aval du bord d'attaque d'un profil plongés dans l'eau, ont été effectuées.

\section{Essais de ventilation}

Les essais de ventilation derrière un seuil de $10 \mathrm{~mm}$ de hauteur pour une vitesse, $V_{0}$, comprise entre 6 et $22 \mathrm{~m} / \mathrm{s}$ ont été effectués au CREMHyG. Les résultats obtenus pour deux vitesses ( 8 et $12 \mathrm{~m} / \mathrm{s}$ ) sont présentés sous la forme d'un débit par unité de largeur en fonction de la longueur de la cavité sur la figure 1. Chaque essai a été reproduit une dizaine de fois de façon à obtenir une estimation de l'incertitude expérimentale associée surtout à l'appréciation de la longueur de la poche. Aux incertitudes près, le débit est proportionnel à la vitesse comme on peut le constater sur la figure 2 où les débits volumiques moyennés divisés par la largeur de la veine et la vitesse (notés $E v$ ) sont portés en fonction de la longueur de la poche. Il faut remarquer qu'il est impossible d'obtenir des cavités de longueur inférieure à $5 \mathrm{~cm}$.

Des essais ont été réalisés à l'ENSTA sur un profil de $15 \mathrm{~cm}$ de corde à extrados plat et à intrados en arc de cercle de rayon $19,5 \mathrm{~cm}$ pour $V_{0}$ variant de 3 à $10 \mathrm{~m} / \mathrm{s}$. Le mode opératoire consiste à imposer un débit d'air et à faire varier l'incidence de façon à obtenir une poche convenablement ventilée sur l'extrados, dont on mesure la longueur. Les résultats, présentés en termes de valeurs moyennes de $E v$, toutes vitesses confondues, ont été portés sur la figure 3 sur laquelle on a aussi superposé les résultats obtenus avec le seuil. Il faut noter que des

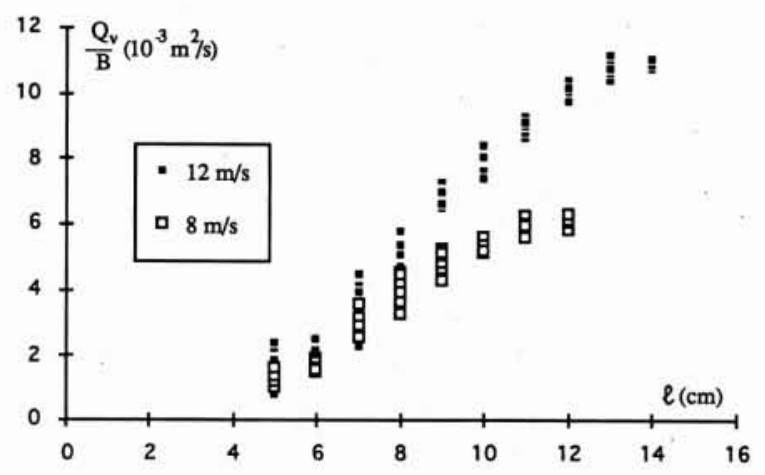

1. Débit volumique d'air injecté par unité de largeur de la veine en fonction de la longueur des cavités. Essais derrière un seuil de $10 \mathrm{~mm}$ de hauteur.

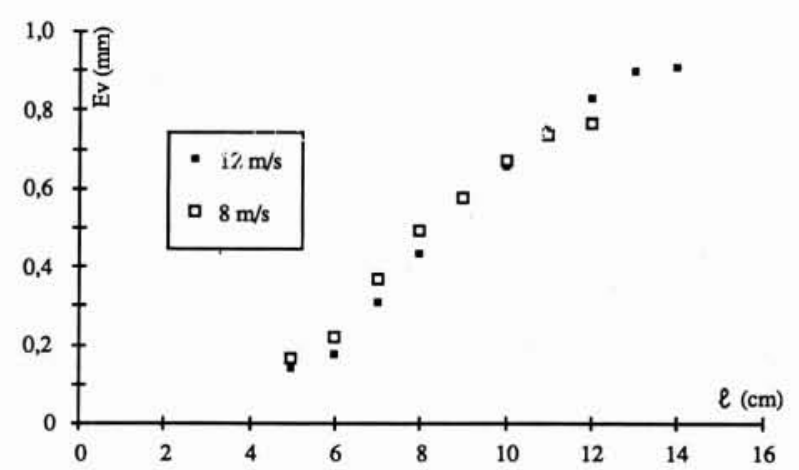

2. Débits volumiques moyennés divisés par la largeur de la veine et la vitesse (notés Ev) en fonction de la longueur des cavités. Essais derrière un seuil de $10 \mathrm{~mm}$ de hauteur.

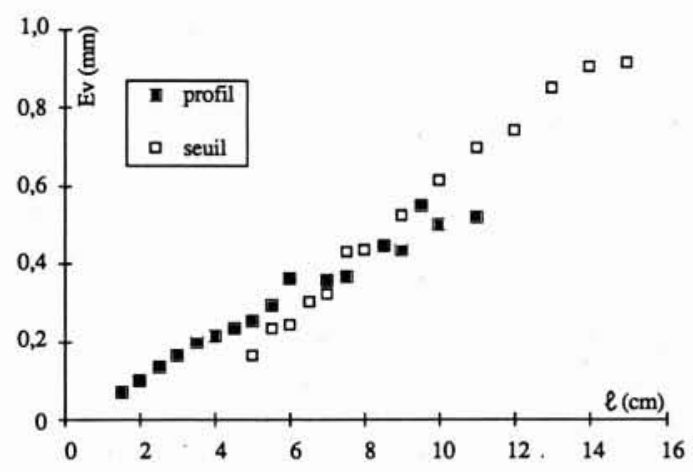

3. Valeurs moyennes de Ev en fonction de la longueur des cavités. Essais derrière un seuil de $10 \mathrm{~mm}$ de hauteur et sur un profil.

poches dont la longueur est supérieure à la demi-corde du profil ne remplissent pas les critères de stabilité et que, de ce fait, les résultats doivent être analysés avec précaution. 
Bien que les géométries des deux obstacles soient très différentes, on constate que, d'une part, les ordres de grandeur de $E v$ sont extrêmement proches dans la zone de recoupement et que, d'autre part, on peut assimiler l'ensemble des résultats à une droite dont la pente est le coefficient de débit,

$$
C_{Q}=\frac{Q_{v}}{B l V_{0}}=\frac{E_{v}}{l}=\frac{v_{g}}{V_{0}}
$$

où $v_{g}$ est la vitesse débitante d'air au travers de la surface de la cavité. En remplaçant dans l'expression (1) le flux de chaleur est donné par,

$$
\Phi_{p}=\rho_{v} C_{Q} B l .
$$

Pour effectuer les calculs on prendra $C_{Q}=5,210^{-3}$.

\section{Comparaison des prédictions avec les résultats de Hord}

HORD (1972) a effectué des mesures de température et de pression à l'intérieur de poches de cavitation attachées sur un profil opérant dans de l'hydrogène liquide, dans de l'azote liquide et sur un venturi dans de l'hydrogène liquide. Pour chacun des essais on connaît les conditions d'entrée $\left(T_{0}, V_{0}\right.$ et $\left.p_{0}\right)$, la longueur de la poche (1) et l'écart maximum entre $T_{0}$ et les températures mesurées sur l'obstacle $\left(\Delta T_{\text {exp }}\right)$. Ces écarts de température vont être comparés à ceux calculés $\left(\Delta T_{\text {calc }}\right)$ pour $x=l$, de manière à obtenir les valeurs maximales, en utilisant une rugosité de $1 \mathrm{~mm}$, valeur qui semble physiquement raisonnable. les valeurs des constantes physiques $\rho_{v}, \rho, \mu, \lambda$, $L$ et $C_{p}$ sont prises à la température $T_{0}$ et à une pression égale à celle de la vapeur à la température $T_{0}$. La formulation utilisée est la suivante,

$$
\begin{aligned}
& \Delta T_{\text {calc }}=2\left(\frac{C_{Q}}{C_{f r}}\right)\left(\frac{L \rho_{v}}{C_{p} \rho}\right)\left[1+2,11(P-1) R_{l}^{-0,1}\right] \\
& \text { avec } \quad P=\frac{\mu}{\lambda} C_{p} \text { et } R_{l}=\frac{\rho V_{0} l}{\mu} .
\end{aligned}
$$

La figure 4 présente les erreurs relatives $\left(\left(\Delta T_{\text {calc }}-\right.\right.$ $\left.\left.\Delta T_{\text {exp }}\right) / \Delta T_{\text {exp }}\right)$ des écarts de température calculées, $\Delta T_{\text {calc }}$, par rapport aux valeurs expérimentales de Hord, $\Delta T_{\text {exp }}$, en fonction du nombre de Reynolds de la cavité pour les trois cas mentionnés ci-dessus. Pour le profil opérant en Hydrogène liquide la moyenne est décalée par rapport au zéro mais l'écart type (bande grisée) est raisonnable puisque légèrement inférieur à $\pm 20 \%$. Dans le cas du Nitrogène liquide, la moyenne est meilleure mais l'écart type est plus élevé, de l'ordre de $30 \%$. Finalement, pour le venturi opérant avec de l'Hydrogène liquide les résultats sont intermédiaires entre ceux des deux liquides utilisés avec le profil. Il faut noter que six points pour lesquels les écarts dépassaient par un ordre de grandeur ceux de tous les autres points ont été supprimés de l'analyse des résultats du venturi.
Il est intéressant de procéder à un regroupement des données pour les trois situations d'écoulement considérées en utilisant une rugosité variable en fonction du nombre de Reynolds et qui a été prise, après quelques tâtonnements mais sans optimisation, égale à $2,2 R_{I}^{-0,5}$. Les résultats sont présentés sur la figure 5 où l'on constate que pour le profil et les deux liquides dans lesquels on a procédé aux essais il existe un bon recouvrement. Le prolongement pour les nombres de Reynolds élevés, supérieurs à $10^{7}$, permis par les essais avec le venturi, est assez raisonnable quoique la dispersion est comparativement plus importante. Les prévisions obtenues en utilisant une modélisation assez simple et rustique permettent d'espérer, grâce au raffinement de quelques paramètres, des améliorations permettant de réduire les erreurs relatives. Il faut néanmoins garder à l'esprit que les résultats de Hord sont entachés d'une imprécision expérimentale, difficile à évaluer, qui est comprise dans les erreurs relatives analysées dans cette section.

\section{Conclusion}

Une méthode semi-empirique permettant la détermination de la température à l'interface d'une cavité de vapeur a été développée. Elle suppose que la quantité de vapeur produite est égale à la quantité d'air qu'il est nécessaire d'injecter pour entretenir une cavité ventilée stable d'égale longueur, quelle que soit la géométrie de l'obstacle sur lequel elle est attachée. En outre, l'interface est modélisée par une plaque plane rugueuse sans gradient de pression au travers de laquelle s'établit un flux de chaleur égal à celui nécessaire pour produire la vapeur. La comparaison des prédictions de cette méthode avec les résultats des expériences de Hord s'avère satisfaisante. Des améliorations tendant à réduire l'erreur relative entre les écarts de température calculés et expérimentaux sont envisagées.

\section{Remerciements}

Ce travail a été effectué grâce au concours financier du Centre National d'Etudes Spatiales. Les auteurs tiennent à exprimer leur gratitude à Monsieur G. Albano, Ingénieur au CNES, pour sa compréhension et ses encouragements. Monsieur Roger Sery et Mademoiselle Ibtissem Benmansour ont été associés à différentes phases de cette étude. 


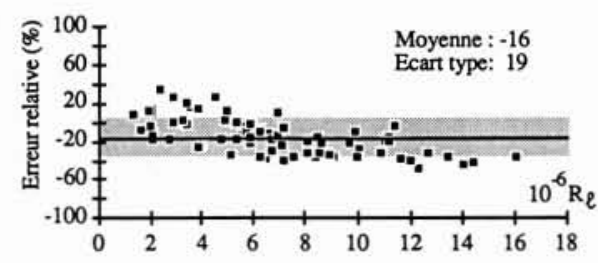

(a)

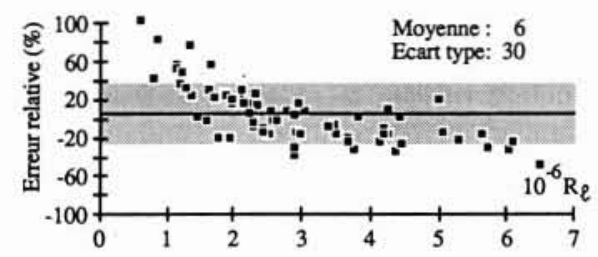

(b)

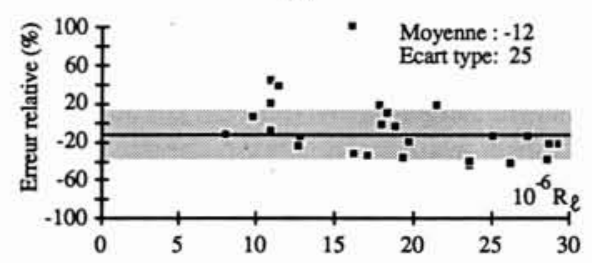

(c)

4. Erreur relative des écarts de température par rapport aux valeurs expérimentales de Hord pour une rugosité constante et : a) le profil dans l' Hydrogène liquide, b) le profil dans le Nitrogène liquide et c) le venturi dans l'Hydrogène liquide.

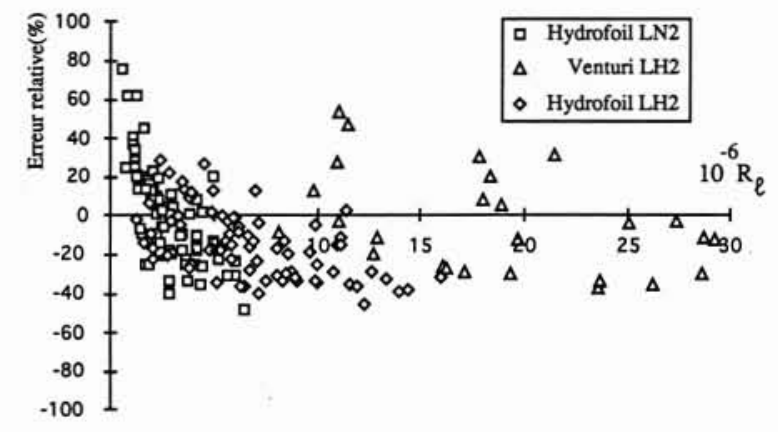

5. Erreur relative des écarts de température par rapport aux valeurs expérimentales de Hord pour une rugosité variable avec le nombre de Reynolds pour l'ensemble des résultats de Hord (profil et venturi).

\section{Références}

BILLet M.L. et WeIR D.S., 1975. - The effect of gas diffusion on the flow coefficient for a ventilated cavity, J. Fluids Eng., 97, p. 501.

BRENNEN C., 1975. - The dynamic balance of dissolved air and heat in natural cavity flows, J. Fluid Mech., 37, p. 115.

Yoshimara K., Kato H., Yamaguchi H. and Miyanaga M., 1988. - Experimental study on the internal flow of a sheet cavity, Cavitation and Multiphase Flow Forum, ASME-FED, vol. 64, pp. 94-98.

Brun E.A., Martinot-Lagarde A. and Mathieu J., 1970. Mécanique des Fluides, 3, Dunod, Paris.

ECKERT E.R.G. et DRAKE R.M., 1972. - Analysis of heat and mass transfer, McGraw-Hill Book Co.
Hord J., Anderson L.M. et Hall W.J., 1972a. - 1972, Cavitation in liquid cryogens : 1-Venturi, NASA CR2054.

HORD J., 1972b. - Cavitation in liquid cryogens : 2-Hydrofoil, NASA CR-2156.

Holl J.W., BILLet M.L. et WeIR D.S., 1975. - Thermodynamic effect on developed cavitation, J. Fluids Eng., 97, p. 507.

MOORE R.D. et RUGGerI R.S., 1969. - Prediction of thermodynamic effects on developed cavitation based on liquid hydrogen and Freon 114 data in scaled venturis, NASA TN D-4899.

Stahl H.A. et Stepanoff A.J., 1956. - Thermodynamics aspects of cavitation in centrifugal pumps, Trans. ASME, J. Basic. Eng., 87, p. 309. 